"Esquisse Historique sur la Marche du Développement de la Géométrie du Triangle," by E. Vigarié; 8vo pamphlet (read before the French Association for the

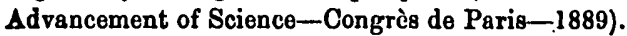

"Sur les Axes de Steiner et l'Hyporbole de Kiepert," et "Sur les Foyers de Steiner d'un Triangle," MMI. J. Neuberg and A. Gob (read before the French Association for the Advancement of Science-Congrès de Paris-1889).

"Sur les Projections et Contre-Projections d'un Triangle Fixe, et Sur le Système de Trois Figures directement Semblables," M. J. Neuberg. (Offprint from Tome xuIv. of the "Mémoires Couronnés et autres Mémoires," publiés par l'Académie Royale de Belgique, 1890.)

\title{
On a Theorem relating to Bicircular Quartics and Twisted
}

Quartics. By R. LACHLAN, M.A.

[Read Iray 8th, 1890.]

The present paper deals with a theorem analogous to the theorem that : Every plane cubic which passes through eight fixed points must pass through a ninth. It will be proved that a very similar theorem exists for plane bicircular quartics, and for twisted quartic curves, and, as might be expected, the theorem leads to as many, if not more, interesting results than the one for the cubic. It has been thought advisable to state for circular curves the corresponding theorems to those which are given in Salmon ("Higher Plane Curves," \$\$ 30-34) for ordinary plane curves, although of course the former may be easily deduced fron these. And it should be stated that a circular curve of the $2 n^{\text {th }}$ order is always understood to mean a curve of order $2 n$, having each of the circular points at infinity as multiple points of the $n^{\text {th }}$ order.

The paper is divided into three sectious: in the first are given the general theory of the intersection of circular curves; in the second section a particular theorem relating to plane bicircular quartics is developed; and in the third section the corresponding theorem for twisted quartics is proved independently, and it is explained how the particular theorems in the second section may be transluted so as to apply to twisted quartics.

\section{General Theorems on Circular Curves, §§ 1-4.}

1. The general equation of a curve of the $2 n^{\text {th }}$ order having multiple points of the $n^{\text {th }}$ order at each of the circular points, is of 
the form

$$
\begin{gathered}
\dot{u}_{0}\left(x^{2}+y^{2}\right)^{n}+u_{1}\left(x^{2}+y^{2}\right)^{n-1}+\ldots+u_{n-1}\left(x^{3}+y^{2}\right) \\
+v_{n}+v_{n-1}+\ldots v_{1}+v_{0}=0,
\end{gathered}
$$

where $u_{r}$ and $v_{r}$ are homogeneons expressions of the $r^{\text {th }}$ degree in $x$ and $y$.

Such a curve is determined when we have given

$$
(1+2+\ldots+n+n+1+n+\ldots+1)-1,
$$

i.e., $\quad n(n+2)$ points.

2. Two circular curves of the $2 m^{\text {th }}$ and $2 n^{\text {th }}$ orders intersect in $2 m n$ points, excluding the circular points. Hence it follows that every circular curre of the $2 n^{\text {th }}$ degree which passes throngh $n(n+2)-1$ fixed points must also pass through $(n-1)^{2}$ other fixed points.

We may also deduce the theorem : If of the $2 n^{9}$ points of intersection of two circular curves each of the $22^{\text {th }}$ order, $2 n p$ lie on a circular curve of order $2 p$, the remaining $2 n(n-p)$ will lie on a circular curve of order $2(n-p)$.

3. Again, every circular curve of the $2 n^{\text {th }}$ order which passes through $2 n p-(p-1)^{2}$ points on a circular curve of order $2 p$ ( $p$ being less than $n$ ) meets this curve in $(p-1)^{2}$ other fixed points.

For through any other $(n-p)(n-p+2)$ points on the curve of order $2 n$ we can draw a circular curve of order $2(n-p)$, and this, with the curve of order $2 p$, makes up a curve of order $2 n$ passing through

$$
2 n p-(p-1)^{2}+(n-p)(n-p+2),
$$

i.e., $n(n+2)-1$ points; hence, by $\S 2$, it must pass through $(n-1)^{2}$ other fixed points on the given curve of order $2 n$; and these must lie on one or other of the curves of orders $2 p$ and $2(n-p)$; but these curves can only meet the given curve in $2 n p$ and $2 n(n-p)$ points, respectively; hence the truth of the theorem is manifest.

As a particular case, we see that every circular curve of the eighth order which passes through 15 points on a bicircular quartic passes through one other fixed point; and every circular curve of the order $2 n$ which passes through $4 n-1$ fixed points on a bicircular quartic must pass through one other fixed point.

4. An extension of the theorem in $\$ 3$ maj be thus stated :Any circular curve of the $2 r^{\text {th }}$ order ( $r$ being greater than $m$ and $n$, T 2 
but less than $m+n-1)$, which passes through all but $(m+n-r-1)^{2}$ of the $2 m n$ points of intersection of two circular curves of orders $2 m$ and $2 n$, will pass also,through the remaining intersections.

For, if we draw a circular curve of order $2(r-m)$ through

$$
(r-m)(r-m+2)
$$

arbitrary points on the curve of order $2 n$; and a circular curve of order $2(r-n)$ through $(r-n)(r-n+2)$ arbitrary points on the curve of order $2 m$; these curves make up, with the curves of orders $2 m$ and $2 n$, two curres each of order $2 r$, and each passing through such a number of the $2 m n$ points of intersection of the given curves of orders $2 m, 2 n$ as make up $r(r+2)-1$ points in each case; and hence these two curves pass throigh $(r-1)^{2}$ other fixed points.

But

$$
\begin{aligned}
r(r+2)-1 & -(r-m)(r-m+2)-(r-n)(r-n-2) \\
& =2 m n-(m+n-r-1)^{2} ;
\end{aligned}
$$

hence the theorem is seen to be true. For the theorem to be applicable, it is easily seen that $r$ must be at least equal to the greater of the integers $m$ and $n$, also $r-m$ must be $<n$, otherwise it would not be possible to draw the auxilialy curve of order $2(r-m)$; and, as the theorem is nagatory when $r=\dot{m}+n-1$, we see that we must have

$$
r<m+n-1 \text {. }
$$

\section{Application to Bicircular Quartics, §§ 5-12.}

5. The general theorem in $\S 3$ states that every circular curve of the eighth degree which passes through 15 fixed points on a bicircular quartic must pass through a sixtcenth fixed point; in other words, if two circular systems each of the eighth degree intersect in 15 points which lie on a bicircular quartic, their sixteenth point of intersection also lies on the quartic.

Suppose we have three circles $A, B, C$ cutting a bicircular quartic respectively in the points $a_{1}, a_{9}, a_{3}, a_{4} ; b_{1}, b_{9}, b_{3}, b_{4} ; c_{1}, c_{2}, c_{3}, c_{4}$; and let the circles $\left(a_{1}, b_{1}, c_{2}\right),\left(a_{2}, b_{2}, c_{2}\right),\left(a_{3}, b_{8}, c_{3}\right),\left(a_{4}, b_{4}, c_{4}\right)$ meet the quartic in the points $d_{1}, d_{2}, d_{3}, d_{4}$, respectively; then these points must be concyclic. For let the circles $\left(a_{1}, b_{1}, c_{1}\right),\left(a_{2}, b_{2}, c_{8}\right),\left(a_{3}, b_{3}, c_{3}\right)$, $\left(a_{4}, b_{4}, c_{4}\right)$ be denoted by $L, M, N, P$; and let $D$ denote the circle $\left(d_{1}, d_{9}, d_{s}\right)$; then each of the systems $A, B, O, D$ and $L, M, N, P$ are of the eighth degree, and fifteen of their points of intersection lie on the quartic, therefore the remaining point in which the system $A, B$, 
$C, D$ meets the quartic must be the point $d_{4}$, which is the sixteenth point in which the system $L, M, N, P$ meets the quartic. Hence the circle $D$ must pass through the point $d_{4}$.

6. Suppose now that the circles $A, B, C$ coincide; then the circles $L, M, N, P$ become the osculating circles at the points $a_{1}, a_{3}, a_{3}, a_{6}$; hence we have the theorem that: The osculating circles at four concyclic points on a bicircular quartic, meet the quartic in fonr points which are also concyclic.

The circle $D$ passing through the points $d_{1}, d_{2}, d_{3}, d_{4}$, in which the osculating circles at $a_{1}, a_{2}, a_{3}, a_{4}$ meet the quartic, might be called the satellite circle of the circle $A$.

7. Let us now suppose the points $a_{1}, a_{2}, a_{8}$ are cyclic points; then the circle $D$ must coincide with the circle $A$; and hence the point $a_{4}$ must either be another cyclic point, or must coincide with one of the points $a_{1}, a_{2}, a_{3}$; in other words, the circle which can be drawn passing through any three cyclic points, meets the quartic in a fourth cyclic point or touches it at one of the three points.

Now we know that there are sixteen cyclic points on a bicircular quartic, viz., four cyclic points on each of the principal circles of the quartic.

Let $a_{1}, a_{2}, a_{3}, a_{4}$ be the four cyclic points on the principal circle $J_{1}$; $\beta_{1}, \beta_{2}, \beta_{3}, \beta_{4}$ the cyclic points on $J_{2} ; \gamma_{1}, \gamma_{2}, \gamma_{3}, \gamma_{4}$ those on $J_{3}$; and $\delta_{1}, \delta_{2}, \delta_{3}, \delta_{4}$ those on $J_{4}$. If $\alpha_{9}$ be the inverse point to $a_{1}$ with respect to $J_{2}$, it is evident that the circles $a_{1} a_{2} \beta_{1}, a_{1} a_{2} \beta_{2}, a_{1} a_{2} \beta_{3}, a_{1} a_{2} \beta_{4}$ will touch the quartic at the points $\beta_{1}, \beta_{2}, \beta_{2}, \beta_{4}$, respectively. And it follows from the theorem that two pairs of inverse points are concyclic, that no other circle passing through $a_{1}, a_{2}$ and a third cyclic point, $\gamma_{1}$ say, touch the curve at either of the points $a_{1}, a_{2}, \gamma_{1}$. Now sixteen points may be arranged in groups of three in 16.15.14/6 $=560$ ways; through each pair of points such as $a_{1}, a_{2}$ we can draw four circles touching the curve at another cyclic point, and we can take a pair such as $a_{1}, a_{2}$ in $6 \times 4$ ways (viz.; 6 pairs on each principal circle); hence, of the 560 circles that are obtained by taking any three of the 16 points, $6 \times 4 \times 4=96$ touch the quartic; we have left 464 circles, and, by what was proved above, each of these must meet the curve in a fourth cyclic point. Dividing this number by 4 , we see that 116 circles can be drawn each passing through four cyclic points on a bicircular quartic.

It will be interesting to see how these circles may be grouped. There are the four principal circles; and then we know that pairs of inverse points are concyclic, thus we shall have 48 circles of the type 
$a_{1} a_{3} \gamma_{1} \gamma_{3}, a_{1}$ and $a_{9}$ being inverse points with respect to $J_{9}$, as also $\gamma_{1}$ and $\gamma_{3}$ : we shall also bave the circles $a_{1} a_{2} \gamma_{2} \gamma_{4}, a_{1} a_{2} \delta_{1} \delta_{3}, a_{1} a_{2} \delta_{8} \delta_{4}$; i.e., four circles for each pair of points $a_{1} a_{2}$ on $J_{1}$, and so $\frac{1}{2} .4 \times 6 \times 4=48$ in all ; the remaining 64 circles will be circles passing through one point on each of the principal circles.

8. We know that nine circles can always be drawn through any point $a$ on a bicircular quartic which will osculate the curve elsewhere; let us suppose, then, that the osculating circles at the points $a_{1}, a_{n}, \ldots a_{0}$ meet the curre again in the point $a$; and let the osculating circles at $b_{1}, b_{8}, \ldots b_{0}$ meet the carre in $b$, the osculating circles at $c_{1}, c_{2}, \ldots c_{0}$ meet in $c$, and the osculating circles at $d_{1}, d_{2}, \ldots d_{0}$ meet the curve in $d$. Then, if the points $a, b, c, d$ are concyclic, it follows from $\S 6$ that the circle passing through three points such as $a_{i}, b_{j}, c_{k}$ must pass through one of the points $d_{1}, d_{2}, \ldots d_{0}$.

Consequently, though there corresponds bat one satellite circle $D$ for every circle $A$, yet corresponding to a circle $D$ there are $9 \times 9 \times 9=729$ different circles $A$.

9. Let us suppose that the points $a, c, d$ coincide; then we see that, if the osculating circle at the point $a$ meets the curve in the point $b$, and if the osculating circles at $a_{1}, a_{2}, \ldots a_{0}$ meet the curve in the point $a$, and the osculating circles at $b_{1}, b_{2}, \ldots b_{8}$ meet the curve in $b$, then the circle passing through any tbree of the points $a_{1}, a_{2}, \ldots a_{0}$ must pass through one of the points $b_{1}, b_{2}, \ldots b_{8}$. In other words, if $a_{1}, a_{2}, \ldots a_{0}$ are the points, the osculating circles at which meet the curve in the point $a$, then the $9.8 .7 / 6=84$ circles which can be drawn through the points $a_{1}, a_{2}, \ldots a_{0}$, taken three at a time, meet again in eight other points which are also on the quartic, and the osculating circles at these eight points meet the curve again in the same point as the osculating circle at the point $a$.

10. Returning to the general theorem in $\S 5$, let as suppose that the circles $A, B, C$ are bitangent circles to the quartic at the points $a, a^{\prime} ; b, b^{\prime} ; c, c^{\prime}$, respectively ; then the circles $L, M$ coincide, and also the circles $N, P$. Hence, if the circles $a b c, a^{\prime} b^{\prime} c^{\prime}$ cut the curre again in the points $d, d^{\prime}$, we infer that the circle which touches the curve at $d$ and passes through $d^{\prime}$ must touch the carve at $d^{\prime}$. If the three bitangent circles $a a^{\prime}, b b^{\prime}, c c^{\prime}$ belong to the same system, i.e., cut orthogonally the same principal circle, then it is obvious that $d, d^{\prime}$ are inverse points with respect to the same principal circle; but in the abore reasoning it would seem that the three bitangent circles $A, B, O$ need not necessarily belong to the same system. 
11. Further, let us suppose that the points $a, b, c$ coincide; then we see that, if three of the bitangent circles at the point $a$ touch the curre again at the points $a_{1}, a_{2}, a_{3}$, respectively, and if the osculating circle at $a$ and the circle $a_{1} a_{2} a_{3}$ meet the curvo again in $d, d^{\prime}$, then $d, d^{\prime}$ are points of contact of a bitangent circle.

Now there are four circles which touch at $a$ and touch the curve elsewhere; let $a_{1}, a_{2}, a_{3}, a_{4}$ be the points of contact (i.e., the inverse points of $a$ with respect to the four principal circles), then we infer that the four circles which can be drawn through these four points meet the curve in four points $d_{1}, d_{2}, d_{3}, d_{4}$ which are the points of contact of the bitangent circles which touch the curve at $d$, the point where the osculating circle at $a$ cuts the curve.

12. Again, let the points of contact of the bitangent circles at $a, b$, $c, d$ be respectively $a_{1}, a_{2}, a_{3}, a_{4} ; b_{1}, b_{2}, b_{3}, b_{4} ; c_{1}, c_{2}, c_{3}, c_{4} ; d_{1}, d_{2}, d_{3}, d_{4}$; then, if $a, b, c, d$ are concyclic, we see, from $\S 10$, that any circle such as $a_{i} b_{j} c_{k}$ must pass through one of the four points $d_{1}, d_{2}, d_{3}, d_{4}$.

Hence these 16 points lie on 64 circles, each circle passing throngh one and only one of the points corresponding to $a, b, c, d$, respectively.

\section{Analogous Theorems in connexion with Troisted Quartics, §§ 13-15.}

13. Since the equation of a bicircular quartic may be written in the form

$$
a x^{2}+b y^{2}+c z^{2}+d w^{2}=0,
$$

where $x, y, z, w$ are the "power-coordinates" of a point referred to four mutually orthogonal circles, so that they are connected by the identical relation

$$
x^{2}+y^{2}+z^{2}+w^{2}=0,
$$

it follows that, corresponding to all theorems connected with bicircular quartics, there exist analogous theorems concerning quartic curves in space. In fact, we have merely to substitute the word "plane" for "circle" in the enunciation of theorems concerning bicircalar quartics to obtain the corresponding theorem for twisted quartics.

'I'hus, in place of the theorem in $\$ 5$, we shall bave the theorem : Every quartic surface which passes through 15 fixed points on the curve of intersection of two quadrics must pass through one other fixed point on the curve.

14. It may be as well to give an independent proof of this theorem. 
Thus, let $u, v$ denote two quadrics, and $S$ any quartic surface passing through 15 fixed points on the curve of intersection of $u$ and $v$; wo have to prove that any other quartic surface which passes through these 15 points must pass through the remaining point of intersection of $S$ with $u$ and $v$.

Now, through any 8 arbitrary points on the curve of intersection of $u$ and $S$, and through an arbitrary point $P$ on $S$, we can draw a quadric, $v^{\prime}$ say ; also, throngh 8 arbitrary points on the curve of intersection of $S$ and $v$, we can draw a quadric, $u$ say, also passing through $P$ ( $P$ being supposed not to lie on either $u$ or $v$ ); then we have three quartic surfaces, $S, u u^{\prime}, \imath v^{\prime}$, each passing through $15+8+8+1=32$ fixed points, and every quartic which passes through these points must be of the form $S+\lambda u t i^{\prime}+\mu v v^{\prime}$, and therefore must pass through the remaining 32 points in which $S, u u^{\prime}$, and $v v^{\prime}$ intersect.

Hence every quartic surface which passes through 15 of the points of intersection of $S, u, v$ must pass through the remaining point of intersection.

15. We have at once the theorem, that if three planes $A, B, C$ be drawn cutting a twisted quartic in the points $a_{1}, a_{2}, a_{3}, a_{6} ; b_{1}, b_{2}, b_{8}, b_{4}$; $c_{1}, c_{2}, c_{8}, c_{4}$, respectively; the planes $a_{1} b_{1} c_{1}, a_{2} b_{2} c_{2}, a_{3} b_{3} c_{3}, a_{4} b_{6} c_{4}$ will cut the quartic in fonr coplanar points $d_{1}, d_{2}, d_{3}, d_{4}$.

Consequently, all the theorems stated previously may be at once translated so as to apply to twisted quartics.

On the Avithmetical Theory of the Form $x^{3}+n y^{3}+n^{2} z^{3}-3 n x y z$. By Professor Mathews, M.A.

[Rend Mray 8th, 1890.]

In the last four papers contrined in Vol. I. of Dirichlet's collected works will be found some remarkable propositions relating to certain arithmetical forms of higher degrees.*

- Dirichlet's Werke. I., pp. $619,625,633,639$. The titles of the papers aro"Bur la Théorio des Nombres" (Comptes Rendus, 1840, p. 285, or Liouville, Sór. I., t. จ., p. 72); " Einige Resultate von Untersuchungen uber oine Olasse homogener Functionen des dritten und der höheren Grade" (Berichte über dio Verhandlungend. Königl. Prewss. Akad. d. Wissensch., 1841, p. 280); "Verallgemeinerung eines 